\title{
New sensitivity goal for neutrinoless double beta decay experiments
}

\author{
Vishnudath K. N., ${ }^{1,2, *}$ Sandhya Choubey, ${ }^{3, \dagger}$ and Srubabati Goswami ${ }^{1, \hbar}$ \\ ${ }^{1}$ Physical Research Laboratory, Ahmedabad 380009, India \\ ${ }^{2}$ Discipline of Physics, Indian Institute of Technology, Gandhinagar 382355, India \\ ${ }^{3}$ Harish-Chandra Research Institute, HBNI, Chhatnag Road, Jhunsi, Allahabad 211 019, India
}

(Received 30 January 2019; published 28 May 2019)

\begin{abstract}
We study the implications of the Dark-LMA (DLMA) solution to the solar neutrino problem for neutrinoless double beta decay $(0 \nu \beta \beta)$. We show that, while the predictions for the effective mass governing $0 \nu \beta \beta$ remain unchanged for the inverted mass scheme, that for normal ordering becomes higher for the DLMA parameter space and moves into the "desert region" between the two. This sets a new goal for the sensitivity reach for the next-generation experiments if no signal is found for the inverted ordering by the future search programs.
\end{abstract}

DOI: 10.1103/PhysRevD.99.095038

\section{INTRODUCTION}

Fermions can be of two types: Dirac or Majorana. A Majorana particle is a self-conjugate fermion. All known fermions other than the neutrino are Dirac particles. Neutrinos, being neutral, are the only known particle that can possibly be a Majorana fermion. Hence, the question whether neutrinos are a Dirac or Majorana particle is one of the most fundamental questions in physics. The most straightforward way to probe the Majorana nature of neutrinos is through neutrinoless double beta decay $(0 \nu \beta \beta)$. While beta decay involves the decay of a radioactive nucleus into a daughter nucleus along with an electron and an electron-type antineutrino, neutrinoless double beta decay is a rare process in which a nucleus of charge $\mathrm{Z}$ and mass number $\mathrm{A}$ decays into a daughter, producing two electrons and no neutrinos: $(A, Z) \rightarrow$ $(A, Z+2)+2 e^{-}$[1]. A positive signal of this will be a definite confirmation of the existence of lepton numberviolating Majorana mass term for the neutrinos [2]. Such a term requires transcending beyond the Standard Model of particle physics and could also be related to the observed preponderance of matter over antimatter, which is essential for our existence. Thus, it is not surprising that searches for $0 \nu \beta \beta$ have been ongoing for the past several decades [3]. While no undisputed positive signal has been seen in any of

\footnotetext{
vishnudath@prl.res.in †sandhya@hri.res.in

*sruba@prl.res.in
}

Published by the American Physical Society under the terms of the Creative Commons Attribution 4.0 International license. Further distribution of this work must maintain attribution to the author(s) and the published article's title, journal citation, and DOI. Funded by SCOAP. the experiments so far, a lower limit (90\% C.L.) on the $0 \nu \beta \beta$ lifetime of $T_{1 / 2}\left({ }^{136} \mathrm{Xe}\right)>10.7 \times 10^{25} \mathrm{yr}$ has been obtained from KamLAND-Zen [4], $T_{1 / 2}\left({ }^{76} \mathrm{Ge}\right)>8 \times$ $10^{25} \mathrm{yr}$ has been obtained from GERDA [5], and $T_{1 / 2}\left({ }^{130} \mathrm{Te}\right)>1.5 \times 10^{25} \mathrm{yr}$ has been obtained from combined results of CURCINO and CUORE [6]. In this work, we assume that $0 \nu \beta \beta$ is driven solely via a Majorana mass term for the neutrinos. Therefore, nonobservation of $0 \nu \beta \beta$ puts an upper limit on the effective neutrino mass, which depends on the neutrino mass and mixing parameters. The effective mass depends crucially on whether the neutrino mass spectrum conforms to normal ordering (NO) or inverted ordering (IO), which corresponds to whether the third mass eigenstate is the heaviest or lightest, respectively. In addition to the neutrino mass ordering, the effective Majorana neutrino mass also depends on the two mass squared differences $\left(\Delta m_{21}^{2}\right.$ and $\left.\Delta m_{31}^{2}\right)$, the mixing angles $\theta_{12}$ and $\theta_{13}$ and the Majorana phases $\alpha_{1}$ and $\alpha_{2}$. By allowing these parameters to vary in their current $3 \sigma$ allowed range, one obtains two bands of predicted values for the effective mass for IO and NO, separated by a "desert region." The effective mass corresponding to IO (approximately $0.015-0.05 \mathrm{eV}$ ) is expected to be probed comfortably in the next-generation $0 \nu \beta \beta$ experiments, which include LEGEND, GERDA-II,MAJORANA D, CUPID, $\mathrm{SNO}+$, KamLAND2-Zen, nEXO, NEXT 1.5K, PANDAX III 1k, SuperNEMO, etc. [7]. While lowering the sensitivity of these experiments to be able to probe the effective mass for the $\mathrm{NO}$ case is going to be challenging, it is possible to make some in roads into this region as well, albeit with a lower probability [8]. Many of these planned experiments will be capable of probing the desert region, i.e., territories $\lesssim 0.01 \mathrm{eV}$, even if they fall short of testing significant parts of the NO band [8-11]. 
In this paper, we show, for the first time, the impact of the so-called Dark-LMA (DLMA) [12-14] solution to the solar neutrino problem on $0 \nu \beta \beta$. The standard LMA solution corresponds to standard neutrino oscillations with $\Delta m_{21}^{2} \simeq$ $7.5 \times 10^{-5} \mathrm{eV}^{2}$ and $\sin ^{2} \theta_{12} \simeq 0.3$ and satisfies the solar neutrino data at high significance. The DLMA solution appears as a nearly degenerate solution to the solar neutrino problem for $\Delta m_{21}^{2} \simeq 7.5 \times 10^{-5} \mathrm{eV}^{2}$ and $\sin ^{2} \theta_{12} \simeq 0.7$, once we allow for the existence of nonstandard neutrino interactions (NSIs) in addition to standard oscillations. The KamLAND experiment is unable to break this degeneracy since it observes neutrino oscillations in vacuum, which depends on $\sin ^{2} 2 \theta_{12}$ which is the same for both the LMA and DLMA solutions. ${ }^{1}$ The occurrence of the DLMA solution can also adversely affect the determination of mass ordering in beam-based neutrino oscillation experiments in the presence of NSIs [15-17]. We will show that, while the IO band for the effective mass in $0 \nu \beta \beta$ experiments remains nearly same for LMA and DLMA solutions, the NO band gets shifted upward for DLMA into the desert region mentioned above. As a result, this may make it possible for the next-generation experiments to start probing $0 \nu \beta \beta$ for NO as well. This entails twofold aspects. First, this opens up unheralded regions of the effective neutrino mass to be probed by future $0 \nu \beta \beta$ experiments. Second, this provides a way of testing the longstanding DLMA solution to the solar neutrino problem, irrespective of the value of the NSI parameters. Scattering experiments can also resolve this degeneracy by measuring the NSI parameters. For instance, in Ref. [18], combined constraints from neutrino oscillation and CHARM and $\mathrm{NuTeV}$ measurements were used to demonstrate that the degeneracy between the two LMA solutions can be resolved if NSI is only with the down quarks. Subsequently, the study performed in Ref. [19] included the COHERENT neutrinonucleus scattering data and showed that the DLMA solution can be disfavored at the $3.1 \sigma$ and 3.6 $\sigma$ C.L. for NSI with up and down quarks, respectively. However, it is worth stressing that these bounds depend on the mass of the light mediator, and it has been shown in Ref. [20] that the COHERENT data exclude the DLMA solution at 95\% C.L. for light mediator mass greater than $48 \mathrm{MeV}$ only. The global analysis including oscillation and COHERENT data performed in Ref. [21] shows that the DLMA solution is still allowed at $3 \sigma$, albeit for a smaller range of values of NSI parameters and for light mediators of mass $\gtrsim 10 \mathrm{MeV}$.

Although the importance of precision determination of $\theta_{12}$ on the effective mass determined by $0 \nu \beta \beta$ experiments has been highlighted earlier [22,23], the ramifications of the DLMA solution for $0 \nu \beta \beta$ is being investigated in this work for the first time.

\footnotetext{
${ }^{1}$ Combining KamLAND and neutrino neutral current scattering experiments like CHARM to lift this degeneracy has been discussed in Ref. [13].
}

\section{PREDICTIONS FOR $0 \nu \beta \beta$}

The half-life for the $0 \nu \beta \beta$ process in the standard threegeneration picture is given as

$$
\frac{\Gamma_{0 \nu \beta \beta}}{\ln 2}=G\left|\frac{M_{\nu}}{m_{e}}\right|^{2} m_{\beta \beta}^{2},
$$

where $G$ contains the phase space factors, $m_{e}$ is the electron mass, and $M_{\nu}$ is the nuclear matrix element (NME). $m_{\beta \beta}$ is the effective neutrino mass given by

$$
m_{\beta \beta}=\left|U_{e i}^{2} m_{i}\right|
$$

$U$ is the unitary Pontecorvo-Maki-Nakagawa-Sakata mixing matrix for the three active neutrinos and is given in the standard parametrization as

$$
U=R_{23} \tilde{R}_{13} R_{12} P,
$$

where $R_{i j}$ are the three rotation matrices defined in terms of the corresponding mixing angles $\theta_{i j}$, with the Dirac $C P$-phase $\delta$ attached to $\tilde{R}_{13}$, and the phase matrix $P=$ $\operatorname{diag}\left(1, e^{i \alpha_{2}}, e^{i\left(\alpha_{3}+\delta\right)}\right)$ contains the Majorana phases. In this paper, we denote the DLMA solution for $\theta_{12}$ in the presence of NSI as $\theta_{D 12}$ and the standard LMA solution as $\theta_{12}$. The $3 \sigma$ ranges of these two parameters are given in Table I $[21,24]$.

In this parametrization, the effective neutrino mass is

$$
m_{\beta \beta}=\left|m_{1} c_{12}^{2} c_{13}^{2}+m_{2} s_{12}^{2} c_{13}^{2} e^{2 i \alpha_{2}}+m_{3} s_{13}^{2} e^{2 i \alpha_{3}}\right|,
$$

where $c_{i j}=\cos \theta_{i j}$ and $s_{i j}=\sin \theta_{i j} .\left|m_{\beta \beta}\right|$ depends on whether the neutrino mass states follow normal or inverted ordering or they are quasidegenerate (QD):

(i) normal ordering: $m_{1}<m_{2} \ll m_{3}$ with

$$
\begin{aligned}
& m_{2}=\sqrt{m_{1}^{2}+\Delta m_{\mathrm{sol}}^{2}} ; \\
& m_{3}=\sqrt{m_{1}^{2}+\Delta m_{\mathrm{sol}}^{2}+\Delta m_{\mathrm{atm}}^{2}}
\end{aligned}
$$

TABLE I. The $3 \sigma$ ranges of different combinations of oscillation parameters relevant for understanding the behavior of the effective mass in different limits.

\begin{tabular}{lccccc}
\hline \hline & $\sin ^{2} \theta_{12}$ & $\sin ^{2} \theta_{D 12}$ & $\cos 2 \theta_{12}$ & $\cos 2 \theta_{D 12}$ & $\sin ^{2} \theta_{13}$ \\
\hline Maximum & 0.350 & 0.725 & 0.45 & -0.30 & 0.024 \\
Minimum & 0.275 & 0.650 & 0.30 & -0.45 & 0.020 \\
\hline \hline
\end{tabular}


(ii) inverted ordering: $m_{3} \ll m_{1} \approx m_{2}$ with

$$
\begin{aligned}
& m_{1}=\sqrt{m_{3}^{2}+\Delta m_{\mathrm{atm}}^{2}} ; \\
& m_{2}=\sqrt{m_{3}^{2}+\Delta m_{\mathrm{sol}}^{2}+\Delta m_{\mathrm{atm}}^{2}}
\end{aligned}
$$

(iii) quasidegenerate: $m_{1} \approx m_{2} \approx m_{3} \gg \sqrt{\Delta m_{\mathrm{atm}}^{2}}$.

Here, $\Delta m_{\mathrm{sol}}^{2}=m_{2}^{2}-m_{1}^{2}$ and $\Delta m_{\mathrm{atm}}^{2}=m_{3}^{2}-m_{2}^{2}\left(m_{1}^{2}-\right.$ $m_{3}^{2}$ ) for NO (IO). Figure 1 shows $m_{\beta \beta}$ as a function of the lightest neutrino mass for both $\mathrm{NO}$ and IO. The pink region is for $\mathrm{NO}$ with the standard solution for $\theta_{12}$, and the red band is for NO with $\theta_{D 12}$, corresponding to the DLMA solution. The dark blue band is for IO with the standard $\theta_{12}$ value, and the cyan band (which overlaps with the blue band) is for IO with $\theta_{D 12}$. The gray band $(0.071-0.161 \mathrm{eV})$ corresponds to the current upper limit from combined results of GERDA and KamLAND-Zen experiments. The region above this is disallowed. The range corresponds to the NME uncertainty $[5,25,26]$. The black dashed line represents the future $3 \sigma$ sensitivity of the $\mathrm{nEXO}$ experiment: $T_{1 / 2}=5.7 \times 10^{27} \mathrm{yr}$ [9], which, for the highest value of NME, translates to $m_{\beta \beta}=0.007 \mathrm{eV}$. This can probe a small part of the NO region with the LMA solution for $m_{\text {lightest }} \gtrsim 0.005 \mathrm{eV}$, whereas the upper edge of the DLMA region can be probed even for small values of $m_{\text {lightest }}$. The yellow region is disfavored by the cosmological constraints on the sum of the light neutrino masses [27]. In obtaining this plot, all the oscillation parameters are

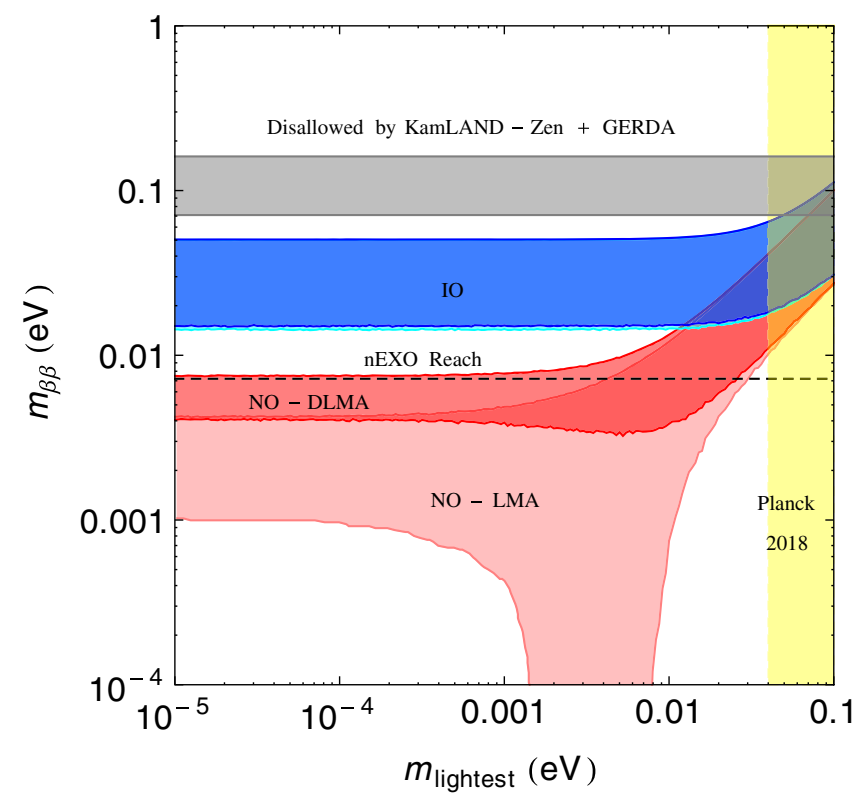

FIG. 1. The effective neutrino mass $m_{\beta \beta}$ for $0 \nu \beta \beta$ as a function of the lightest neutrino mass for both $\mathrm{NO}$ and IO. The pink region is for $\mathrm{NO}$ with the standard solution for $\theta_{12}$, and the red band is for NO with $\theta_{D 12}$. For the IO case (the blue band), $m_{\beta \beta}$ remains the same for the DLMA solution. See the text for details. varied in their $3 \sigma$ ranges [24], and the Majorana phases are varied from 0 to $\pi$.

From the figure, we can see that for $\mathrm{NO} m_{\beta \beta}$ for the DLMA solution is higher than that for the standard LMA solution, shifting into the gap between IO and NO. The effect is more pronounced for lower values of $m_{\text {lightest }}$. There is some overlap in the predictions between the maximum value of $m_{\beta \beta}$ for the LMA with the minimum value of this for the DLMA solution, which increases as $m_{\text {lightest }}$ increases. One noteworthy feature is the absence of the cancellation region for the DLMA solution. For IO, the predicted values of $m_{\beta \beta}$ remain the same for LMA and DLMA solutions. Since the predictions of $m_{\beta \beta}$ for NO with LMA and IO with DLMA are well separated, the generalized hierarchy degeneracy [16] is not present.

The behavior of $m_{\beta \beta}$ can be understood by considering the limiting cases for different mass schemes.

Inverted ordering: In this case, for very small values of $m_{3}$ such that $m_{3} \ll \sqrt{\Delta m_{\mathrm{atm}}^{2}}, m_{2} \approx m_{1} \approx \sqrt{\Delta m_{\mathrm{atm}}^{2}}$, the effective mass is given as

$$
m_{\beta \beta \mathrm{IO}} \approx \sqrt{\Delta m_{\mathrm{atm}}^{2}}\left(\left|c_{12}^{2} c_{13}^{2}+s_{12}^{2} c_{13}^{2} e^{2 i \alpha_{2}}\right|\right) .
$$

In this region, $m_{\beta \beta}$ is independent of $m_{3}$ and is bounded from above and below by a maximum and minimum value given by [28],

$$
\begin{aligned}
& m_{\beta \beta_{\mathrm{IO} \text { max }}}=\left|c_{13}^{2} \sqrt{\Delta m_{\mathrm{atm}}^{2}}\right| \quad\left(\alpha_{2}=0, \pi\right), \\
& m_{\beta \beta_{\mathrm{IO} \text { min }}}=\left|c_{13}^{2} \cos 2 \theta_{12} \sqrt{\Delta m_{\mathrm{atm}}^{2}}\right| \quad\left(\alpha_{2}=\pi / 2\right) .
\end{aligned}
$$

The maximum value is independent of $\theta_{12}$, while for the minimum value, we can see from Table I that the $3 \sigma$ range for $\left|\cos 2 \theta_{12}\right|$ is the same for both the LMA and DLMA solutions. This explains why the prediction for $m_{\beta \beta}$ is the same for both the cases in this region.

Now, as $m_{3}$ approaches approximately $\sqrt{\Delta m_{\text {atm }}^{2}}$, the other masses can be approximated as $m_{1} \approx m_{2} \approx$ $\sqrt{2 \Delta m_{\mathrm{atm}}^{2}}$, and the effective mass becomes

$$
m_{\beta \beta \mathrm{IO}}=\sqrt{\Delta m_{\mathrm{atm}}^{2}}\left|\left(\sqrt{2} c_{13}^{2}\left(c_{12}^{2}+s_{12}^{2} e^{2 i \alpha_{2}}\right)+s_{13}^{2} e^{2 i \alpha_{3}}\right)\right| .
$$

This is maximum for $\alpha_{2}=\alpha_{3}=0$ and is again independent of $\theta_{12}$. Also, $m_{\beta \beta_{\mathrm{IO}}}$ is minimum for $\alpha_{2}=\pi / 2$ and $\alpha_{3}=0$ or $\pi / 2$ depending on whether we take $\theta_{12}$ or $\theta_{D 12}$. But since $s_{13}^{2}$ is very small, this is almost independent of what we choose for $\alpha_{3}$, and, effectively, the minimum of $m_{\beta \beta_{\mathrm{IO}}}$ in this regime is approximated as

$$
m_{\beta \beta \mathrm{IO} \text { min }}=\sqrt{\Delta m_{\mathrm{atm}}^{2}}\left|\sqrt{2} c_{13}^{2} \cos 2 \theta_{12}\right|,
$$

which is independent of the solution for $\theta_{12}$. 
Normal ordering: Unlike in IO, the behavior of $m_{\beta \beta}$ is different for the LMA as well as the DLMA solutions of $\theta_{12}$. For very small values of $m_{1}$ such that $m_{1} \ll$ $m_{2} \approx \sqrt{\Delta m_{\mathrm{sol}}^{2}} \ll m_{3} \approx \sqrt{\Delta m_{\mathrm{atm}}^{2}}, m_{\beta \beta}$ can be written as

$$
m_{\beta \beta N 0}=\sqrt{\Delta m_{\mathrm{atm}}^{2}}\left|\sqrt{r} s_{12}^{2} c_{13}^{2} e^{2 i \alpha_{2}}+s_{13}^{2} e^{2 i \alpha_{3}}\right|,
$$

where $r=\left|\frac{\Delta m_{\text {sol }}^{2}}{\Delta m_{\text {atm }}^{2}}\right|$. The maximum value of this corresponds to $\alpha_{2}=\alpha_{3}=0, \pi$, and the minimum value corresponds to $\alpha_{2}=0$ and $\alpha_{3}=\pi / 2$. These will be higher for higher values of $\sin ^{2} \theta_{12}$. This explains why the prediction for $m_{\beta \beta}$ for the DLMA solution in this region is higher.

Moving on to the cancellation region, the typical values of masses are $m_{1} \sim 0.005 \mathrm{eV}, m_{2} \sim 0.01 \mathrm{eV}$, and $m_{3} \sim 0.05 \mathrm{eV}$. Then, the minimum of $m_{\beta \beta}\left(\alpha_{2}=\alpha_{3}=\right.$ $\pi / 2$ ) can be approximated as

$$
m_{\beta \beta \min } \approx m_{1}\left|\left(1-3 s_{12}^{2} c_{13}^{2}-11 s_{13}^{2}\right)\right| .
$$

For the values of $s_{12}^{2}$ and $s_{13}^{2}$ as listed in the Table I, complete cancellation is possible in the LMA region. However, for $s_{12}^{2}$ in the DLMA region, such a cancellation is not possible because of higher values of $s_{12}^{2}$.

As we increase the value of $m_{1}$ and reach the limit of partial hierarchy where $m_{1} \approx m_{2} \approx \sqrt{\Delta m_{\text {sol }}^{2}} \ll m_{3} \approx$ $\sqrt{\Delta m_{\mathrm{atm}}^{2}}$, the maximum value of $m_{\beta \beta}$ is given by

$$
m_{\beta \beta_{\mathrm{NO} \max }} \approx \sqrt{\Delta m_{\mathrm{atm}}^{2} r} c_{13}^{2} \quad\left(\alpha_{2}=\alpha_{3}=0\right),
$$

which is independent of $\theta_{12}$. Hence, the maximum values of $m_{\beta \beta}$ for the two LMA solutions tend to overlap. In the QD limit, $m_{\beta \beta}$ varies linearly with the common mass scale $m_{0}$, and both maximum and minimum values are independent of $\theta_{12}$.

At this point, it is worthwhile to note that if we assume the existence of a fourth sterile neutrino as suggested by the LSND/MiniBooNE results then even for NO the predicted $m_{\beta \beta}$ can be in the desert region [29,30]. In fact, depending on the value of the mass squared difference governing the LSND/MiniBooNE oscillations, the prediction can even overlap with the IO prediction for three generations and hence can be probed by the near-future experiments.

\section{SENSITIVITY IN THE FUTURE EXPERIMENTS}

Here, we discuss a simple method to obtain the sensitivity of the DLMA region in the future ${ }^{136} \mathrm{Xe}$ experiments following the discussion in Ref. [8]. The discovery sensitivity is prescribed as the value of $T_{1 / 2}$ for which an experiment has a $50 \%$ probability of measuring a $3 \sigma$ signal above the background. It is defined as

$$
T_{1 / 2}=\ln 2 \frac{N_{A} \epsilon}{m_{a} S_{3 \sigma}(B)} .
$$

Here, $N_{A}$ is the Avogadro number; $m_{a}$ is the atomic mass of the isotope; $B=\beta \epsilon$ is the expected background where $\epsilon$ and $\beta$ denote the sensitive exposure and background, respectively; $S_{3 \sigma}$ is the value for which half of the measurements would give a signal above $B$ assuming a Poisson signal and is calculated from the relation

$$
1-C D F_{\text {Poisson }}\left(C_{3 \sigma} \mid S_{3 \sigma}+B\right)=50 \% .
$$

$C_{3 \sigma}$ denotes the number of counts for which the cumulative Poisson distribution with mean $B$ follows $C D F_{\text {Poisson }}\left(C_{3 \sigma} \mid B\right)=3 \sigma$. To avoid the discrete variations that would arise in the discovery sensitivity if $C_{3 \sigma}$ is restricted to be integer valued, we use the following definition of $C D F_{\text {Poisson }}$ as a continuous distribution in $C$ using the normalized upper incomplete gamma function:

$$
C D F_{\text {Poisson }}(C \mid \mu)=\frac{\Gamma(C+1, \mu)}{\Gamma(C+1)} .
$$

Using the above equations, the $T_{1 / 2}$ discovery sensitivities of ${ }^{136} \mathrm{Xe}$ as a function of $\epsilon$ for various values of $\beta$ are shown in Fig. 2. In this plot, the red shaded band corresponds to the new allowed region of $m_{\beta \beta} \sim 0.004-0.0075 \mathrm{eV}$ for the DLMA solution. This band in $m_{\beta \beta}$, which is due to the variation of the parameters in the Pontecorvo-MakiNakagawa-Sakata matrix, is converted to a band in $T_{1 / 2}$ using Eq. (1), by taking into account the NME uncertainty as given in Table II. The pink band corresponds to

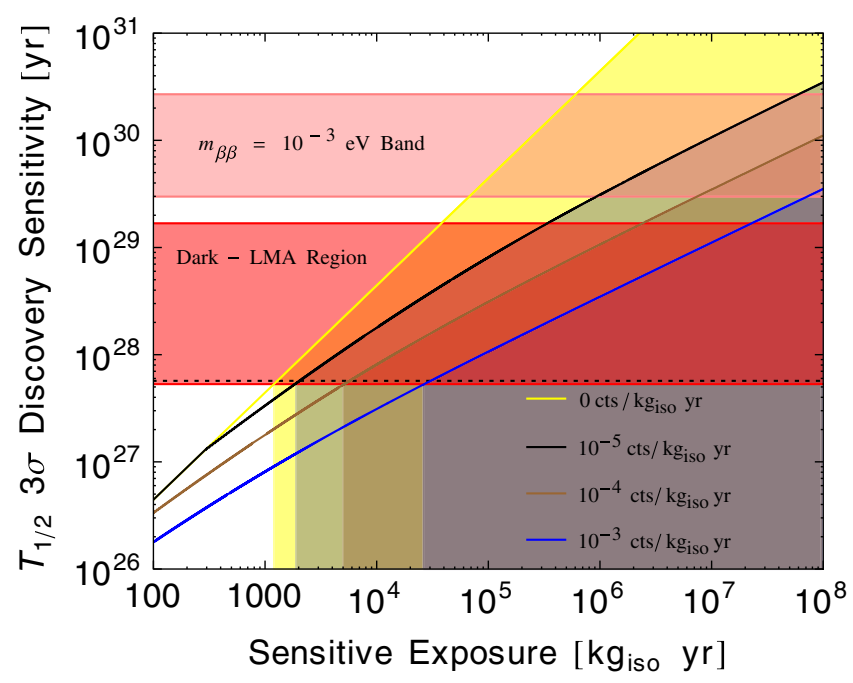

FIG. 2. ${ }^{136} \mathrm{Xe}$ discovery sensitivity as a function of sensitive exposure for a selection of sensitive background levels. The yellow, black, brown and blue lines correspond to different values of the sensitive background levels of $0,10^{-5}, 10^{-4}$ and $10^{-3}$ cts $/\left(\mathrm{kg}_{\text {iso }} \mathrm{yr}\right)$ respectively. 
TABLE II. The $T_{1 / 2}$ ranges corresponding to the DLMA region $m_{\beta \beta}=0.004-0.0075 \mathrm{eV}$ for different isotopes. The NME values $[5,25]$ and the phase space factors [26] used in the calculation are also given.

\begin{tabular}{lccc}
\hline \hline Isotope & $\operatorname{NME}\left(M_{\nu}\right)$ & $G\left(10^{-15} \mathrm{yr}^{-1}\right)$ & $T_{1 / 2}$ range $(\mathrm{yr})$ \\
\hline${ }^{136} \mathrm{Xe}$ & $1.6-4.8$ & 14.58 & $5.3 \times 10^{27}-1.7 \times 10^{29}$ \\
${ }^{76} \mathrm{Ge}$ & $2.8-6.1$ & 2.363 & $2.0 \times 10^{28}-3.4 \times 10^{29}$ \\
${ }^{130} \mathrm{Te}$ & $1.4-6.4$ & 14.22 & $4.9 \times 10^{28}-2.2 \times 10^{29}$ \\
\hline \hline
\end{tabular}

$m_{\beta \beta}=10^{-3} \mathrm{eV}$, which is the minimum of the NO regime for lower values of $m_{\text {lightest }}$ with the LMA solution. In Fig. 2, the dotted black line corresponds to the future $3 \sigma$ sensitivity of nEXO, which is $T_{1 / 2}=5.7 \times 10^{27} \mathrm{yr}$ [9]. The yellow, black, brown, and blue lines correspond to different values of the sensitive background levels of 0 , $10^{-5}, 10^{-4}$, and $10^{-3} \mathrm{cts} /\left(\mathrm{kg}_{\text {iso }} \mathrm{yr}\right)$, respectively. From the figure, we can see that for a sensitive background level of $10^{-4} \mathrm{cts} /\left(\mathrm{kg}_{\text {iso }} \mathrm{yr}\right)$ the DLMA region could be probed with a sensitive exposure greater than approximately $5000 \mathrm{~kg}_{\text {iso }}$ yr. To probe the $10^{-3}$ regime shown by the dashed lines requires lower background levels and/or higher sensitive exposure. In Table II, we have given the $T_{1 / 2}$ ranges corresponding to the DLMA region, $m_{\beta \beta}=0.004-0.0075 \mathrm{eV}$, for three different isotopes.

\section{CONCLUSION}

Searching for the $0 \nu \beta \beta$ process is of utmost importance since it can establish the Majorana nature of the neutrinos, which implies they are their own antiparticles. This will in turn signify a lepton number-violating Majorana mass term for the neutrinos, which may hold the key to explaining why neutrino masses are much smaller than the other fermion masses. This can have profound implications for a deeper understanding of physics beyond the Standard Model of particle physics. So far, these searches have yielded negative results and have put an upper bound on the effective mass governing $0 \nu \beta \beta$. Assuming light Majorana neutrino exchange as the sole mechanism for $0 \nu \beta \beta$, the predictions of effective mass for IO and NO are separated by a desert region. The current upper bound is just above the IO region (approximately $0.1 \mathrm{eV}$ ), and several future experiments with a sensitivity reach of approximately $0.015 \mathrm{eV}$ are expected to probe the IO parameter space completely. However, if no positive signal is found in these searches, then the projected sensitivity reach of these experiments is in the ballpark of $0.005 \mathrm{eV}$, which can explore only a small part of the NO region for lightest neutrino mass $\gtrsim 0.005 \mathrm{eV}$ [9]. The next frontier that is envisaged is approximately $10^{-3} \mathrm{eV}$ [31]. In this paper, we show for the first time that if the DLMA solution to the solar neutrino problem is true then the effective mass for NO shifts into the intermediate desert zone between NO and IO. Therefore, in an incremental advancement, a new goal for the $0 \nu \beta \beta$ experiments can be to first explore this region approximately $0.004-0.0075 \mathrm{eV}$, which is possible even for very low values of the lightest neutrino mass. This not only defines a newer sensitivity goal of the future $0 \nu \beta \beta$ experimental program for the NO scenario but can also provide an independent confirmation/refutal of the DLMA solution to the solar neutrino problem in the presence of nonstandard interactions.

\section{ACKNOWLEDGMENTS}

The authors would like to thank the organizers of the IITB-ICTP workshop on neutrino physics, during which this idea was generated, and Frank Deppisch, K. N. Deepthi and T. Poddar for useful comments.
[1] W. H. Furry, Phys. Rev. 56, 1184 (1939).

[2] J. Schechter and J. W. F. Valle, Phys. Rev. D 22, 2227 (1980).

[3] A. S. Barabash, Phys. At. Nucl. 74, 603 (2011).

[4] A. Gando et al. (KamLAND-Zen Collaboration), Phys. Rev. Lett. 117, 082503 (2016); 117, 109903(A) (2016).

[5] M. Agostini et al. (GERDA Collaboration), Phys. Rev. Lett. 120, 132503 (2018).

[6] C. Alduino et al. (CUORE Collaboration), Phys. Rev. Lett. 120, 132501 (2018).

[7] S. Dell'Oro, S. Marcocci, M. Viel, and F. Vissani, Adv. High Energy Phys. 2016, 2162659 (2016).

[8] M. Agostini, G. Benato, and J. Detwiler, Phys. Rev. D 96, 053001 (2017).
[9] S. A. Kharusi et al. (nEXO Collaboration), arXiv: 1805.11142 .

[10] J. Myslik (LEGEND Collaboration), arXiv:1810.00849.

[11] D. R. Artusa et al. (CUORE Collaboration), Eur. Phys. J. C 74, 3096 (2014).

[12] O. G. Miranda, M. A. Tortola, and J. W. F. Valle, J. High Energy Phys. 10 (2006) 008.

[13] F. J. Escrihuela, O. G. Miranda, M. A. Tortola, and J. W. F. Valle, Phys. Rev. D 80, 105009 (2009); 80, 129908(E) (2009).

[14] Y. Farzan and M. Tortola, Front. Phys. 6, 10 (2018).

[15] P. Bakhti and Y. Farzan, J. High Energy Phys. 07 (2014) 064.

[16] P. Coloma and T. Schwetz, Phys. Rev. D 94, 055005 (2016). 
[17] K. N. Deepthi, S. Goswami, and N. Nath, Phys. Rev. D 96, 075023 (2017).

[18] P. Coloma, P. B. Denton, M. C. Gonzalez-Garcia, M. Maltoni, and T. Schwetz, J. High Energy Phys. 04 (2017) 116.

[19] P. Coloma, M. C. Gonzalez-Garcia, M. Maltoni, and T. Schwetz, Phys. Rev. D 96, 115007 (2017).

[20] P. B. Denton, Y. Farzan, and I. M. Shoemaker, J. High Energy Phys. 07 (2018) 037.

[21] I. Esteban, M. C. Gonzalez-Garcia, M. Maltoni, I. MartinezSoler, and J. Salvado, J. High Energy Phys. 08 (2018) 180.

[22] S. Choubey and W. Rodejohann, Phys. Rev. D 72, 033016 (2005).

[23] A. Dueck, W. Rodejohann, and K. Zuber, Phys. Rev. D 83, 113010 (2011).
[24] I. Esteban, M. C. Gonzalez-Garcia, A. HernandezCabezudo, M. Maltoni, and T. Schwetz J. High Energy Phys. 01 (2019) 106.

[25] J. Engel and J. Menndez, Rep. Prog. Phys. 80, 046301 (2017).

[26] J. Kotila and F. Iachello, Phys. Rev. C 85, 034316 (2012).

[27] N. Aghanim et al. (Planck Collaboration), arXiv:1807 .06209 .

[28] S. Pascoli and S. T. Petcov, Phys. Lett. B 544, 239 (2002).

[29] S. Goswami and W. Rodejohann, Phys. Rev. D 73, 113003 (2006).

[30] J. Barry, W. Rodejohann, and H. Zhang, J. High Energy Phys. 07 (2011) 091.

[31] J. T. Penedo and S. T. Petcov, Phys. Lett. B 786, 410 (2018). 